\title{
Aggregation and chirality
}

Haoke Zhang, Ryan T. Kwok, Jacky W. Lam, Ben Zhong Tang

Haoke Zhang, Ryan T. K. Kwok, Jacky W. Y. Lam, Ben Zhong Tang,

"Aggregation and chirality," Proc. SPIE 10735, Liquid Crystals XXII, $107350 \mathrm{H}$ (14 September 2018); doi: 10.1117/12.2324632

Event: SPIE Organic Photonics + Electronics, 2018, San Diego, California, United States 


\title{
Aggregation and Chirality
}

\author{
Haoke Zhang a,b, Ryan T. K. Kwok ${ }^{\mathrm{a}, \mathrm{b}}$, Jacky W. Y. Lam ${ }^{\mathrm{a}, \mathrm{b}}$, Ben Zhong Tang ${ }^{\text {a,b,c }}$ \\ a Department of Chemistry, Hong Kong Branch of Chinese National Engineering Research Center for \\ Tissue Restoration and Reconstruction, The Hong Kong University of Science and Technology, \\ Clear Water Bay, Kowloon, Hong Kong, China; ${ }^{b}$ HKUST-Shenzhen Research Institute, No. 9 \\ Yuexing 1st RD, South Area, Hi-tech Park Nanshan, Shenzhen 518057, China; ${ }^{\mathrm{c} N F S C}$ Center for \\ Luminescence from Molecular Aggregates, SCUT-HKUST Joint Research Institute, State Key \\ Laboratory of Luminescent Materials and Devices, South China University of Technology, \\ Guangzhou 510640, China; *Corresponding Author: tangbenz@ust.hk
}

\begin{abstract}
Debate on the origin of chirality has lasted for several centuries. Lots of theories in different fields have been propounded to illustrate the possible sources. However, there were no convincing proofs could be provided. In this work, through constructing the relationship between aggregation and chirality, a significant insight was brought to explain how the chirality was generated from the achiral system. Besides, aggregation-induced circular dichroism (AICD) effect was discovered in some AIEgens decorated with amino acid or sugars and aggregation-annihilated circular dichroism (AACD) effect was reported in axially chiral systems. In AICD systems, it was proved that the chirality could transfer from chiral centers to AIEgens in the aggregate state but not solution. Meanwhile, it was concluded that the right-handed helical structures corresponded to a negative Cotton effect and vice versa. For the AACD effect, further studies suggested that the annihilation was caused by the change of twist angle within the process of aggregation.
\end{abstract}

Keywords: Spontaneous resolution, aggregation-induced circular dichroism (AICD), aggregation-annihilated circular dichroism (AACD)

\section{INTRODUCTION}

Chirality is an old and mysterious research topic. It is no exaggeration to say that chirality is an essential element of the life on earth as all the amino acids, proteins and deoxyribonucleic acid (DNA) are chiral. Also, the chirality of these molecules shows an important effect on the physiological activity. However, as an academic term, the chirality was first put forward in 1884 which was quite close to us. Actually, little is known about where does the chirality come from in nature. For example, why the amino acids mostly exist as $L$-enantiomer but sugars are $D$-enantiomer in the human body? Why is most of the deoxyribonucleic acid (DNA) right-handed? Currently, several theories were proposed as the possible mechanisms which may induce the chirality from achiral systems. Such as parity non-conservation theory caused the mirror symmetry breaking, asymmetric catalysis by circularly polarized light and evolution generated the enantiomeric separation. ${ }^{1}$ However, no direct evidence could be provided to fully support these hypotheses.

Liquid Crystals XXII, edited by lam Choon Khoo, Proc. of SPIE Vol. 10735, 107350H

(c) 2018 SPIE · CCC code: 0277-786X/18/\$18 - doi: 10.1117/12.2324632 
Recently, Daniel et al. from NASA Goddard Space Flight Center discovered the $L$-amino acid enrichment in two different fragments of the $\mathrm{C} 2$ Tagish Lake meteorite. ${ }^{2}$ They also demonstrated that the aqueous alteration on the meteorite parent body played an important role in the $L$-excess. Similarly, the amino acids in the human body also experienced the change of external aqueous environment at all time, which may cause the spontaneous resolution. For example, the amino acid could be soluble in one microenvironment. However, driven by the metabolism, the amino acid might transfer to another insoluble microenvironment. The process of aggregation should have a great effect on the enantiomeric separation.

In this field, a series of works have been carried out to clarify the relationship between aggregation and chirality. Some of these systems focused on how the aggregation effect facilitated the enantiomeric separation from the achiral system, the others studied the variation of chiroptical properties within the process of aggregation. Part of these works was summarized at here and some general conclusions were drawn to illustrate how the aggregation effect can affect the chirality.

\section{FROM ACHIRAL MOLECULE TO CHIRAL AGGREGATE}

Tetraphenylethylene (TPE) was a well-studied molecule with aggregation-induced emission (AIE) effect which was coined by Tang et al. in 2001. ${ }^{3}$ Materials with AIE effect usually possessed propeller-shaped structures (Figure 1) which showed no emission in solution but its aggregates had a quite strong one. Further study in this area revealed that the working mechanism of AIE was the restriction of intramolecular motion (RIM), which suggested that the nonradiative decay pathway could be blocked once the molecular motion was restrained. ${ }^{4}$ As shown by the chemical structures of these AIE luminogens (AIEgens), they could exhibit chiral properties if the phenyl rings in each molecule were arranged in one direction $(P$ or $M)$. Indeed, the intrinsic chirality from TPE have been verified by Zhang et al. ${ }^{5,6}$ They obtained two kinds of crystals from the different solvent. X-Ray diffraction results showed that one of them had $P$ conformation and the other one was M. Meanwhile, their chiroptical signal was opposite to each other. All of these results suggested that the propellershaped AIEgens showed intrinsic chirality. However, the chiral properties only existed in regular crystal state as the racemization would be generated once the crystal was dissolved.
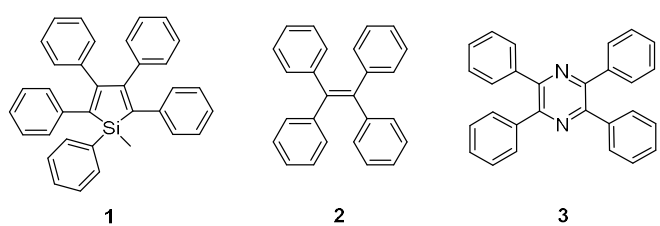

3

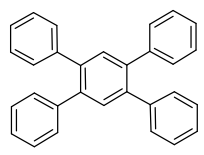

4

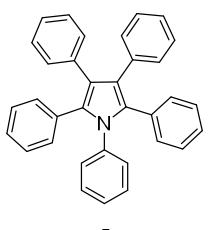

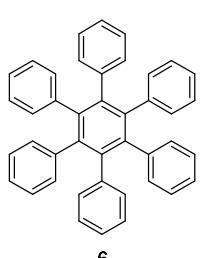

6

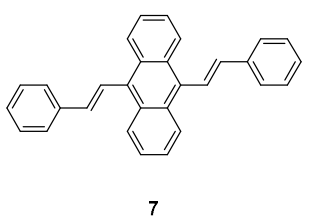

Figure 1. Chemical structures of luminogens with aggregation-induced emission effect. 
Bhosale et al. reported the abnormal chiroptical properties of molecule 8 which was a TPE derivative. ${ }^{7}$ As shown in Figure $2 \mathrm{~A}$, no circular dichroism (CD) signal was detected in hexane/THF (v/v, 1/9) mixture. It was believed that the two enantiomers $P-\mathbf{8}$ and $M-\mathbf{8}$ had $0 \%$ enantiomeric excess (ee), that was the reason why the good solvent mixture showed the achiral property. However, a strong CD signal was observed once the hexane was replaced with a poor solvent of water. The intensity was even higher at methanol/THF (v/v, 1/9) and acetonitrile/THF (v/v, 1/9) mixtures. These results suggested that the addition of poor solvent into the THF solution could induce the enantiomeric separation, which was quite similar to the cases mentioned in the introduction part. But, what was the driving force for the spontaneous resolution? Scanning electron microscope (SEM) was used to investigate its microstructures and the results indicated that helical structures were formed in methanol/THF mixture. The formation of left-handed helical structure could be the source of chiroptical properties. Then, the authors gave a hypothetic illustration of the self-assembly process with the driving force of hydrogenbond and $\pi-\pi$ interaction (Figure 2D), which suggested the formation of right-handed helical superstructures was preferred.
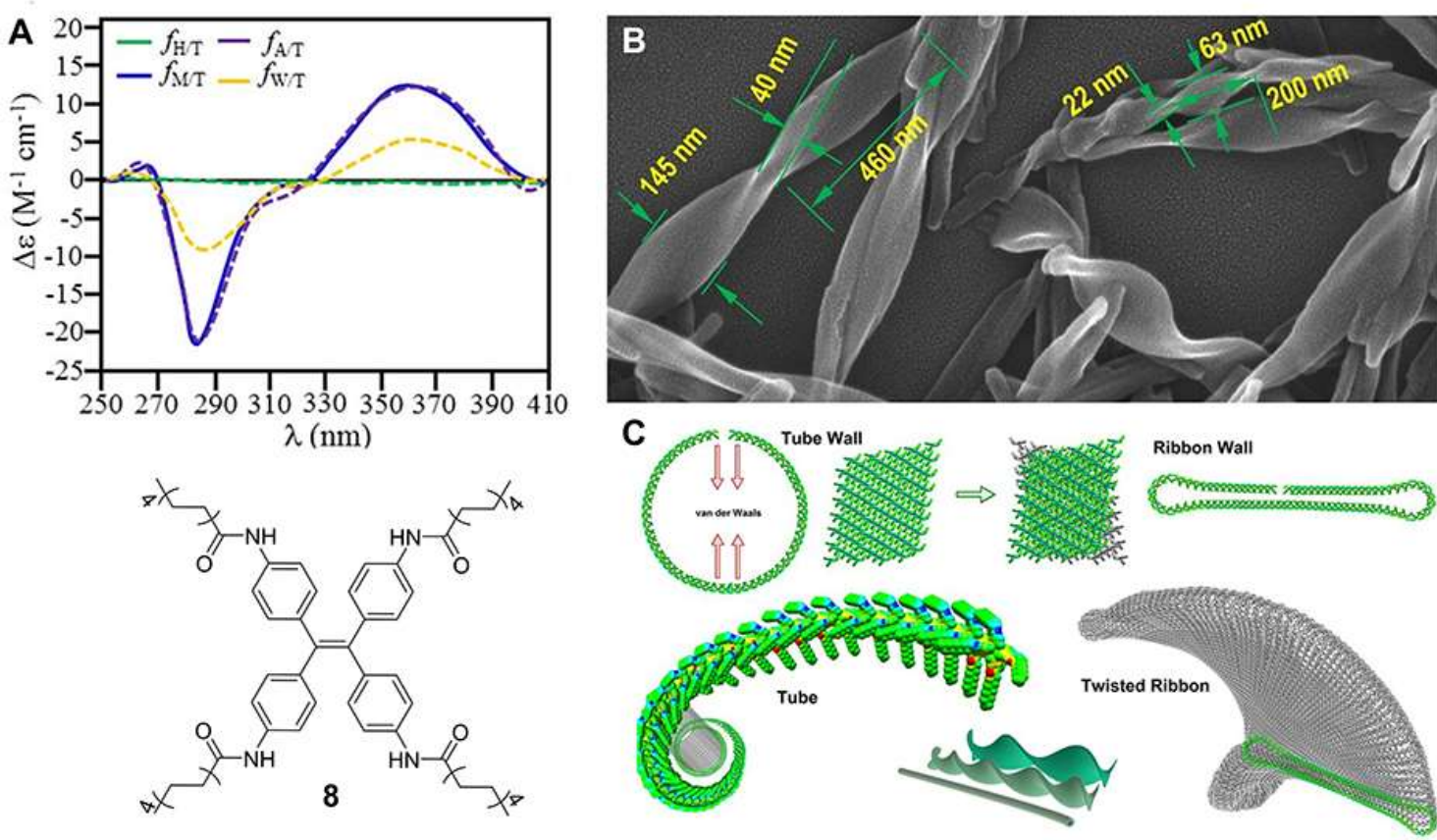

Figure 2. A) CD spectra of 8 in hexane/THF (v/v, 1/9), methanol/THF (v/v, 1/9), acetonitrile/THF (v/v, 1/9) and H ${ }_{2} \mathrm{O} / \mathrm{THF}(\mathrm{v} / \mathrm{v}, 1 / 9)$ mixtures. B) SEM image of the film prepared from methanol/THF mixture. C) Hypothetic illustration of right-handed helical superstructures. Copyright (C) 2015 Springer Nature.

However, it seems that there are several problems with this work. One is that a confused definition of the right-handed helical structure was used. For example, the helical structures in SEM image should be left-handed but the cartoon in Figure $2 \mathrm{C}$ demonstrated the formation of right-handed helical structure. Another question is that authors ascribed the preferred form of right-handed structures to the difference of the solubility between trans- and cis-isomers. However, there was no evidence to support the existence of trans- and cis-geometries in TPE symmetric derivative 8.

Recently, Tang et al have reported another interesting system TPP-Cage (Figure 3). Tetraphenylpyrazine (TPP) was also an archetypical AIEgen which was similar to TPE. The middle benzene in TPE provided stronger intramolecular steric 
hindrance than TPE. Interestingly, the resultant amphiphilic TPP-cage exhibited strong CD signal in acetonitrile without any further chiral separation. The CD intensity increased for more than ten times when changed the solvent from acetonitrile to acetonitrile/water (v/v, 5/95) mixture. Actually, previous works have already proved that the racemization in TPE could be prohibited if four phenyl rings were locked. ${ }^{8,9}$ Figure $3 \mathrm{C}$ showed the rotation barrier calculation for TPPCage, which also suggested the strong intramolecular steric hindrance in the cage. So, it was acceptable that TPP-Cage possessed stable chirality even dissolved in its good solvent. However, it was quite strange that such a symmetric reaction could generate an ee value bigger than $0 \%$. Meanwhile, why the aggregation could facilitate the spontaneous resolution?
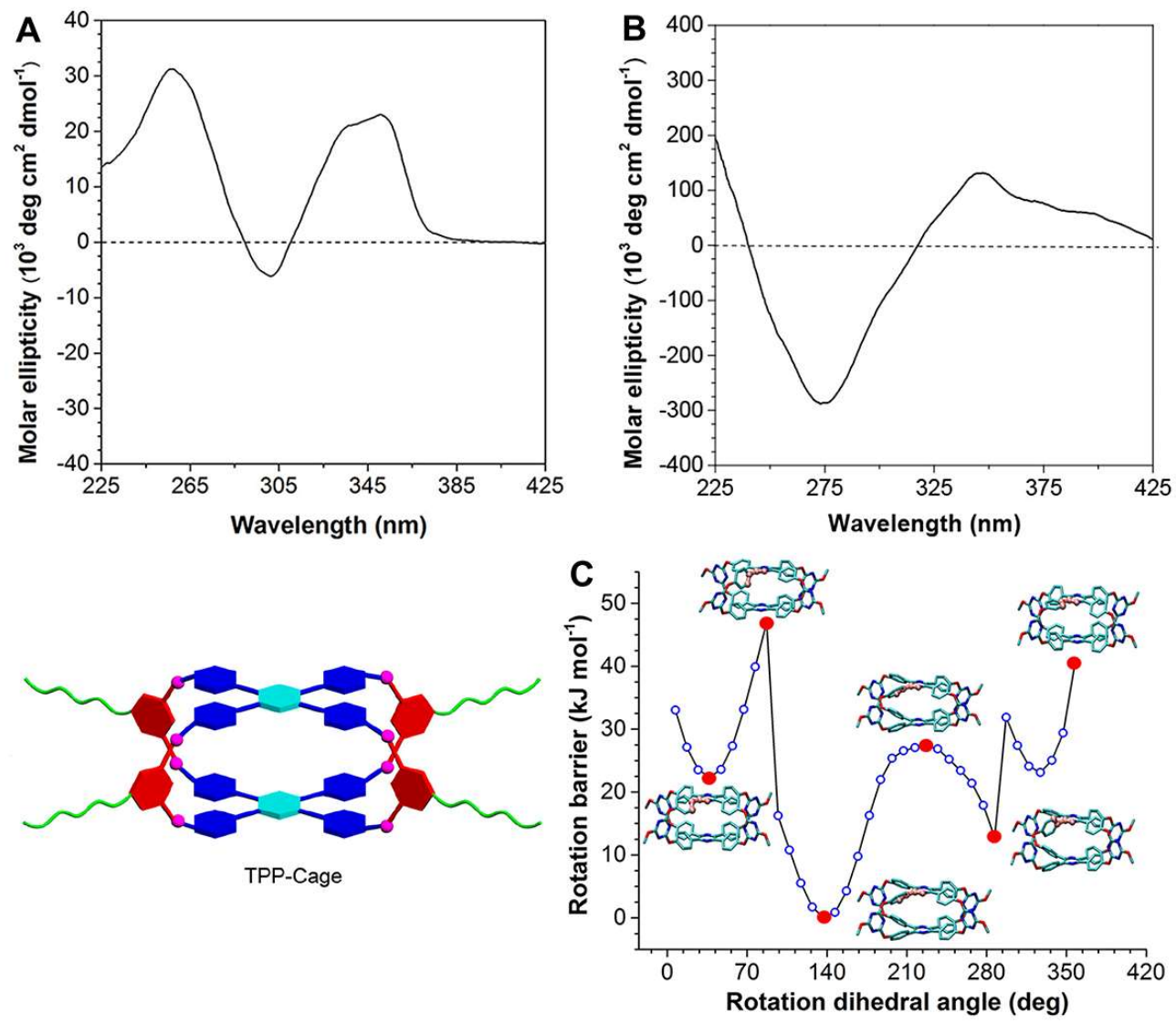

Figure 3. CD spectra of TPP-Cage in A) acetonitrile and B) acetonitrile/water (v/v, 5/95). C) Potential energy surface of TPP-Cage. Copyright (C) 2017 American Chemical Society.

The chiroptical property of hexaphenylsilole (HPS) was reported by the same group. ${ }^{10}$ Figure 4 showed that there was no $\mathrm{CD}$ or circularly polarized luminescence (CPL) signal when HPS was dissolved in its good solvent THF. However, a positive cotton effect and negative CPL signal were obtained in its cast film prepared from THF solution. Meanwhile, these kind of signal were repeatable but not a probability event. After that, the microstructure of HPS in the cast film was also investigated. Both AFM and TEM results suggested the formation of left-handed fibers. Coincidentally, the above mentioned TPE derivative $\mathbf{8}$ also preferred to form left-handed helical superstructures. Besides, almost same CD spectra were recorded for HPS and $\mathbf{8}$ which showed positive cotton effect. 

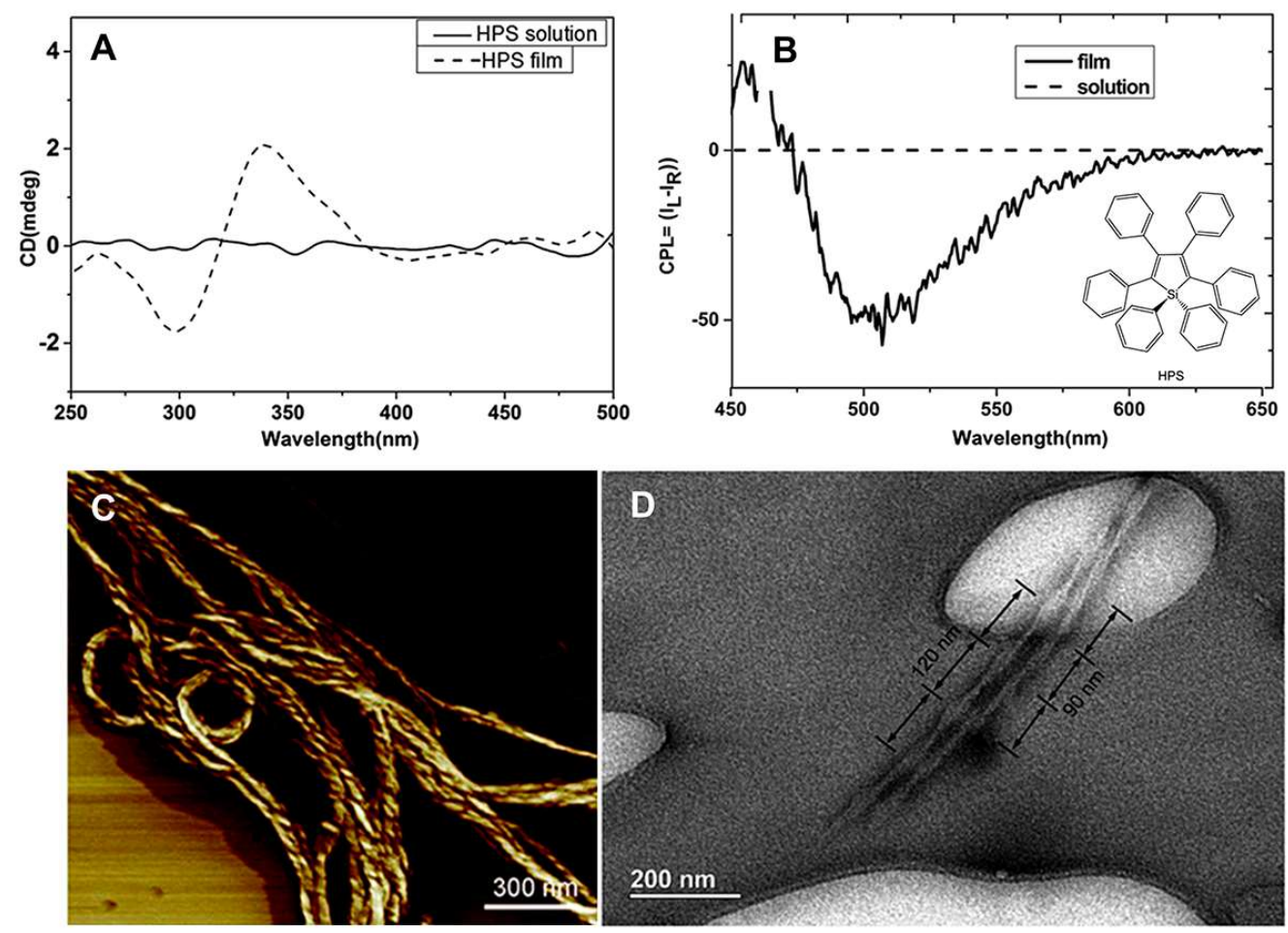

Figure 4. A) CD and B) CPL spectra of HPS in THF solution and film state. C) AFM and D) TEM images of HPS prepared from THF/water (1/4) mixture. Copyright (C) 2017 Royal Society of Chemistry.

In summary, several works have reported the generation of the chirality form achiral system. Among the rest, aggregation has been proved to play an important impact in the spontaneous resolution. Some groups ascribed the resolution to the difference of solubility between two isomers. Others believed that the chirality came from the preferred form of left-handed helical structures. Although all of these results could not make people fully convinced, it brought significant insight into the origin of chirality.

\section{FROM CHIRAL MOLECULE TO CHIRAL AGGREGATE}

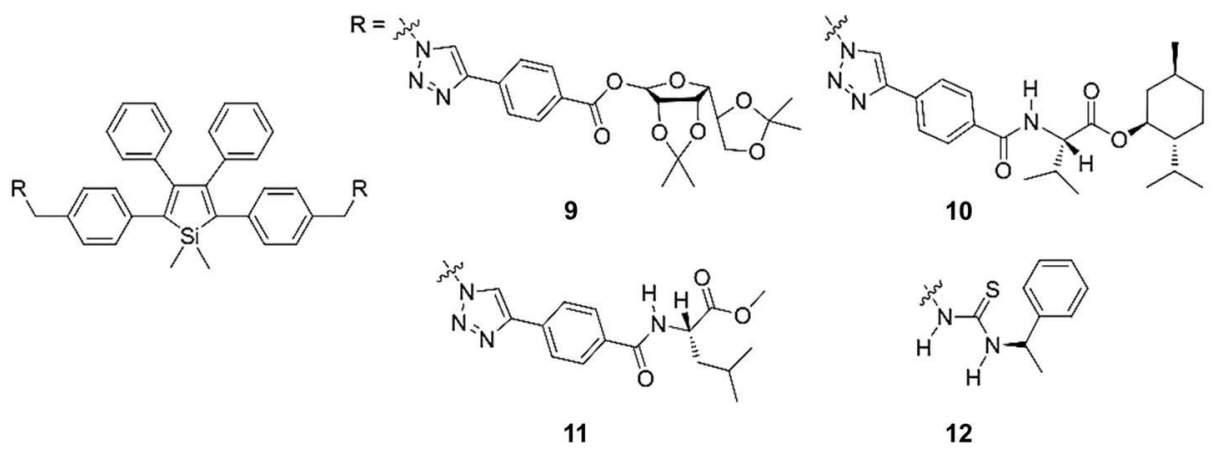

Figure 5. Chemical structures of chiral silole derivatives. 
Fundamentally, unraveling the origin of chirality was quite important for promulgating the laws of nature. From the viewpoint of application, building chiral materials with efficient chiroptical properties was also significant and urgent. As materials, its properties were highly dependent on the chemical structures. Also, the morphology and external environment have a great impact on the performance. Based on this, a lot of chiral molecules with AIE effect were designed and synthesized as the AIE feature gave excellent solid-state photophysical properties to these materials. The design idea was decorating AIEgens with chiral compounds, such as amino acid and sugar. It was expected that the chirality could transfer from chiral sugar to achiral AIEgens.
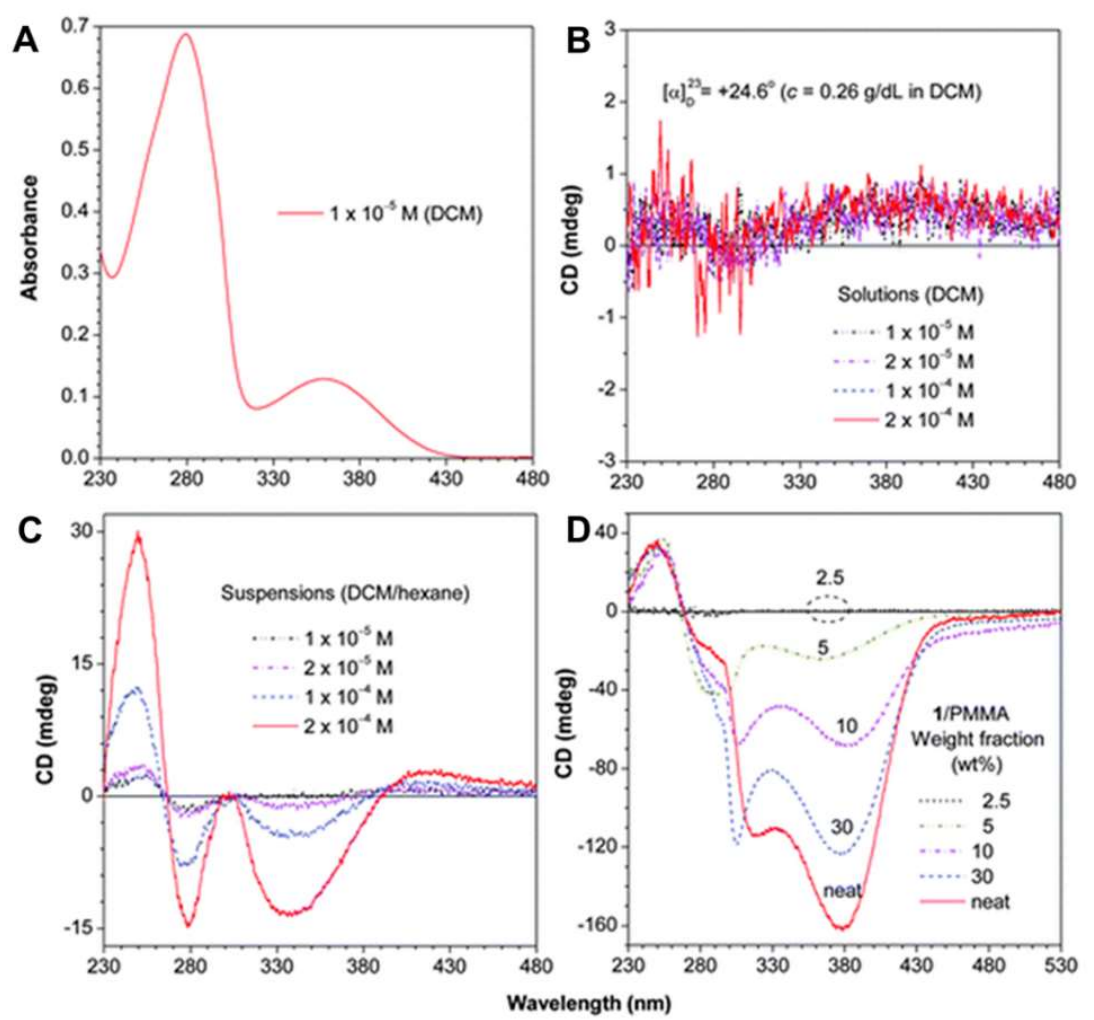

Figure 6. A) UV and B) CD spectra of 9 in dichloroethane (DCE). C) CD spectra of 9 in DCE/hexane (1/9, v/v) mixture with different concentration. D) CD spectra of 9 with different weight fractions (wt\%) dispersed in PMMA matrix. Copyright (C) 2012 Royal Society of Chemistry.

Figure 5 showed a series of chiral silole derivatives developed by Tang et al. ${ }^{11-14}$ For example, two mannoses were decorated at two sides of silole and the molecule 9 was obtained. Figure $6 \mathrm{~A}$ indicated that the maximum absorption $\left(\lambda_{\mathrm{abs}}\right)$ of 9 was located around $360 \mathrm{~nm}$ in dichloroethane (DCE). However, no CD signal was detected for the same solution and even increasing the concentration from 10 to $200 \mu \mathrm{M}$, which suggested that the chirality could not transfer from mannose to silole in pure DCE solution (Figure 6B). Interestingly, the CD signal was induced with a negative cotton effect once added the poor solvent into DCE $\left(\mathrm{V}_{\mathrm{DEC}} / \mathrm{v}_{\text {hexane }}=1 / 9\right)$. Meanwhile, the CD intensity was enhanced with the increase of concentration. Doping compound 9 in poly(methyl methacrylate) (PMMA) matrix with different weight fraction showed the same effect with the suspensions. The CD intensity was enhanced with the increase of weight fraction of 9. But the 
maximum CD wavelength in film exhibited a $40 \mathrm{~nm}$ bathochromic-shift. These results suggested that the chirality could transfer from mannose to the core of silole in the aggregate state. This effect was termed as aggregation-induced circular dichroism (AICD).
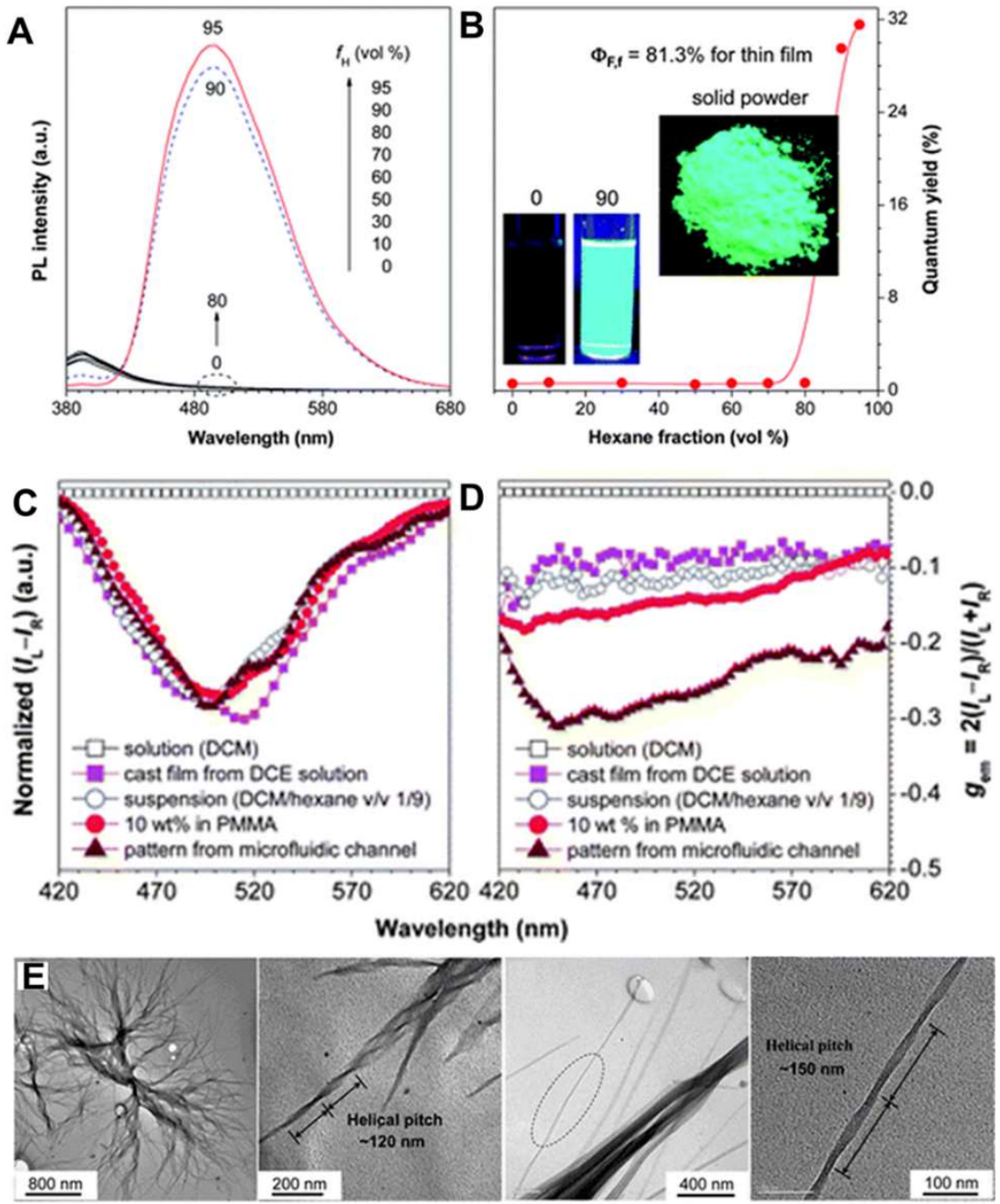

Figure 7. A) PL spectra and B) fluorescence quantum yields of 9 in DCE/hexane mixture with different hexane fraction. C) CPL spectra and D) CPL dissymmetry factor $\left(g_{\mathrm{em}}\right)$ of 9 in different formats: DCM solution, neat cast film from DCE solution of $2 \mathrm{mg} \mathrm{mL}^{-1}, \mathrm{DCM}^{-}$ hexane (v/v, 1/9) mixture (suspension), dispersion in polymer matrix (10 wt \% in PMMA), and fabricated micropattern by evaporating of DCE solution in microfluidic channels. E) TEM images of the aggregates of 9. Copyright (C) 2012 Royal Society of Chemistry.

As expected, compound 9 showed typical AIE effect and its solid-state fluorescence quantum yield $\left(\Phi_{\mathrm{F}}\right)$ was as high as 81.3\% (Figure 7A\&B). Similar with the CD property, no CPL signal was detected in pure DCE solution and strong ones were observed in the aggregate state. Especially for the micropattern fabricated by evaporating of DCE solution in microfluidic channels, a -0.32 CPL dissymmetry factors $\left(g_{\mathrm{em}}\right)$ was achieved, whose value was two orders of magnitude higher than those of commonly reported organic materials. TEM images clearly showed the formation of right-handed helical fibers, which should be the inducer of chiral transfer in the aggregate state. Comparing 9 with HPS, the direction 
of their helical structures was the opposite. Meanwhile, their CD spectra also exhibited the opposite Cotton effect. So, for AIEgens, it could preliminarily conclude that the right-handed helical structures corresponded to a negative Cotton effect and vice versa.<smiles>[R]Cc1ccc(C(=C(c2ccccc2)c2ccccc2)c2ccccc2)cc1</smiles><smiles>[R]Cc1ccc(C(=C(c2ccccc2)c2ccccc2)c2ccc(C[R])cc2)cc1</smiles>

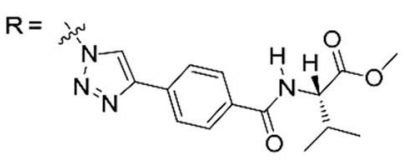

13

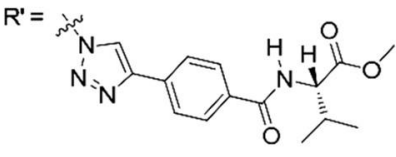

15

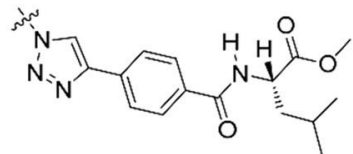

14

Figure 8. Chemical structures of chiral TPE derivatives.

In order to popularize this method, a series of chiral derivatives based on TPE core were also synthesized (Figure 8). ${ }^{15-20}$ Theoretically, they should have the same chiroptical properties with above-mentioned silole derivatives. The absorption spectrum of 13 indicated a $320 \mathrm{~nm} \lambda_{\mathrm{abs}}$ in pure THF solution (Figure 9A). Figure 9B showed that there was no CD signal when molecule 13 was dissolved in THF. Similarly, CD signal with a positive Cotton effect was induced in DCE/hexane mixture with $90 \%$ hexane fraction $\left(f_{\mathrm{H}}\right)$. The intensity was dramatically enhanced in the cast film prepared from THF solution. CPL spectrum was also recorded for the same film with a $0.03 \mathrm{~g}_{\mathrm{em}}$, whose value was one order higher than the traditional pure organic materials. Analysis of the microstructure indicated the formation of left-handed helical superstructures (Figure 9E-H). As expected, it seems that the left-handed helical structures corresponded to a positive Cotton effect, which further proved that the above conclusions were reliable.
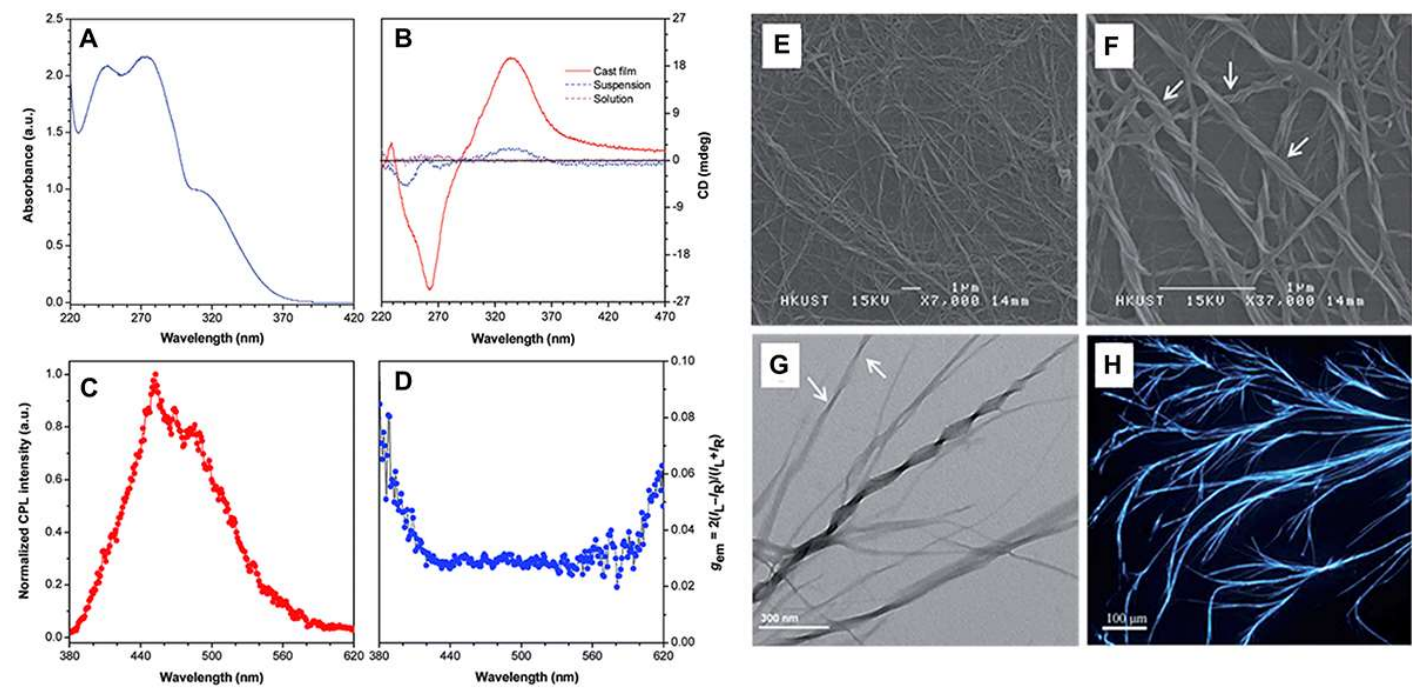

Figure 9. A) UV spectrum of $\mathbf{1 3}$ in THF. B) CD spectra in DCE, DCE/hexane (v/v, 1/9) mixture and cast film. C) CPL and D) $g_{\text {em }}$ spectra in the cast film. E) and F) SEM images, G) TEM image of 13 prepared from DCE/hexane (v/v, 1/9) mixture. H) Fluorescence microscope image of 13, fabricated micropattern by evaporating of DCE solution in microfluidic channels. Copyright (C) 2014 Royal Society of Chemistry. 
In this part, the AICD effect was proposed, which indicated the chirality could transfer from amino acid or sugar to the AIEgens once the aggregates were formed. ${ }^{21-22}$ However, the transfer cannot happen in the solution as the helical superstructures were absent in this state, which suggested that the aggregation played an important role in the chiral transfer. Meanwhile, it was concluded that, for AIEgens, the formation of left-handed helical structures corresponded to a positive Cotton effect and vice versa.

\section{FROM CHIRAL MOLECULE TO ACHIRAL AGGREGATE}

Above mentioned chiral AIEgen derivatives in part three were built on the chiral centers. There is another kind of important chiral system - axial chirality. Does the AICD effect still be suitable for the axially chiral AIEgens? In order to answer this question, Tang et al. synthesized three chiral AIEgens $(R)-\mathbf{1 6},(R)-\mathbf{1 7}$ and $(S)-\mathbf{1 7}$ which bridged two TPE with one 1,1binaphthyl $(\mathrm{BN})$ over Suzuki coupling reaction (Figure 10). ${ }^{23} \mathrm{BN}$ is a famous compound with strong and stable axial chirality. It was expected that the chirality could also transfer from BN to TPE.

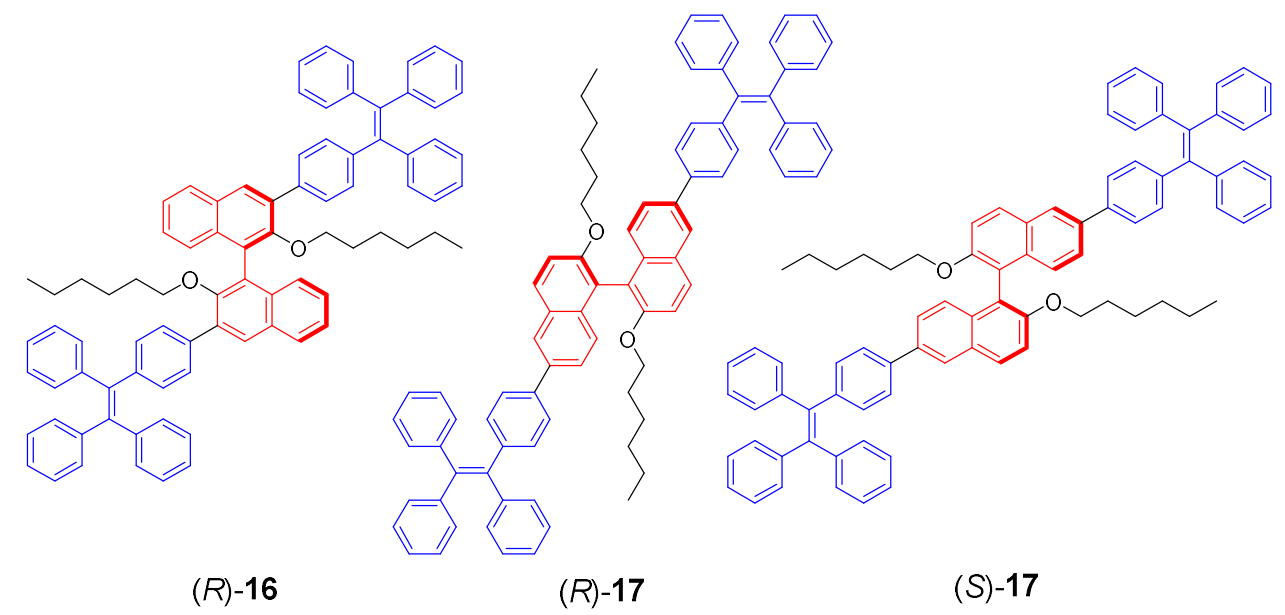

Figure 10. Chemical structures of $(R)-\mathbf{1 6},(R)-17$ and $(S)$-17.

As suggested by Figure 11, $(R)-\mathbf{1 6},(R)-\mathbf{1 7}$ and $(S)$-17 already showed strong CD signal in their good solvent THF, which suggested that the axial chirality transferred from BN to TPE even in the free state which was different with the AICD system. However, the $\mathrm{CD}$ intensity gradually decreased with the increase of water fraction. At aggregate state, the $\mathrm{CD}$ signal was almost annihilated which showed the opposite phenomenon to AICD effect, this effect was termed as aggregation-annihilated circular dichroism (AACD) by Tang et al. Morphology study showed that only the solid spherical particles were observed both in solution and aggregate states, which indicated that the chirality of these three molecules was independent on the molecular self-assembly. So, which kind of effect caused the annihilation? Previous studies reported that the $\mathrm{CD}$ intensity of $\mathrm{BN}$ was highly dependent on the twist angle between two adjacent naphthalene rings in one $\mathrm{BN}$ molecule. ${ }^{24-27}$ Is it possible that the annihilation was caused by the twist angle change of BN units? 

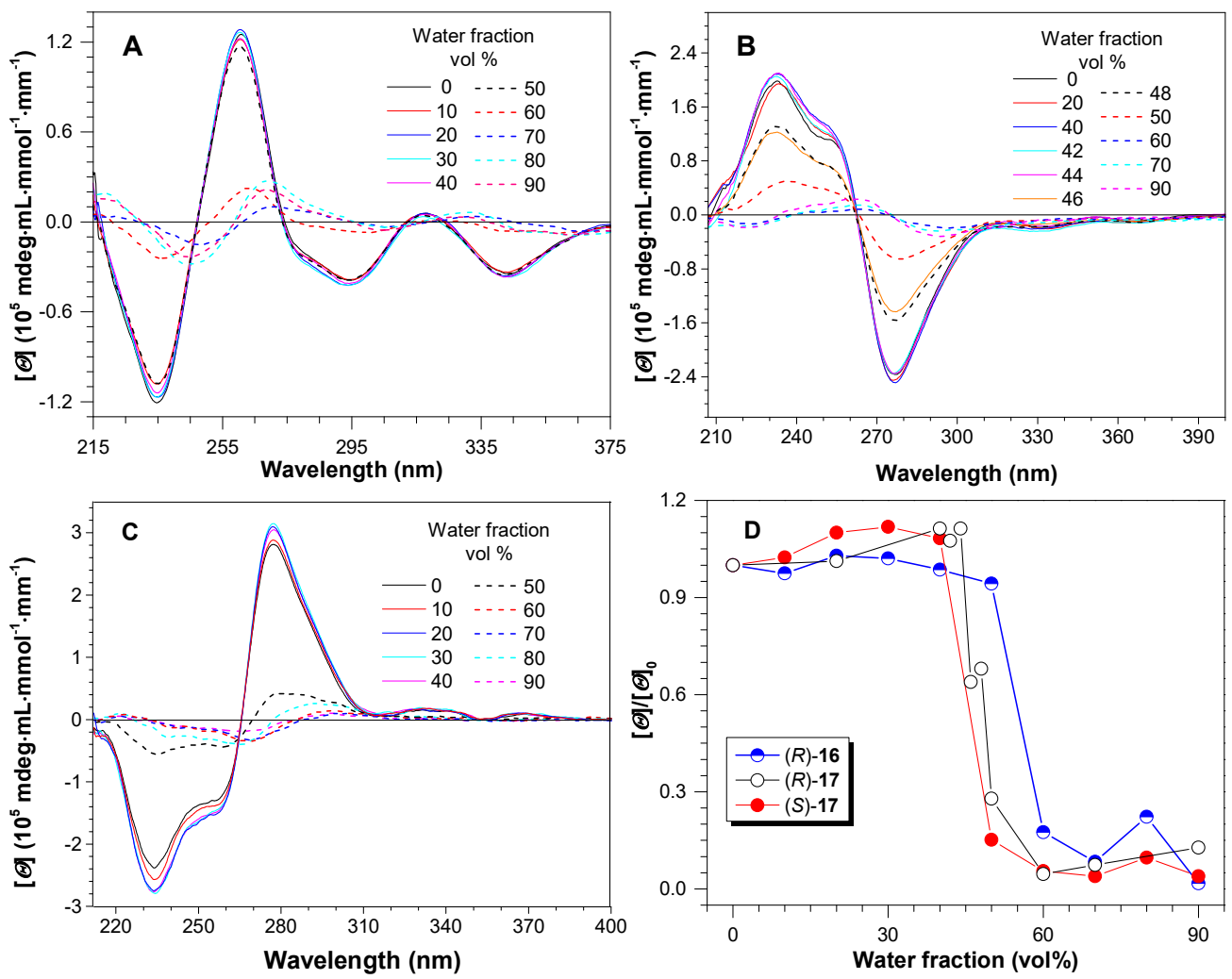

Figure 11. CD spectra of A) $(R)-\mathbf{1 6}, \mathrm{B})(R)-\mathbf{1 7}$ and C) $(S)-\mathbf{1 7}$ in THF/water mixture with different water fraction $\left(f_{\mathrm{w}}\right)$. (D) Plots of $[\Theta] /[\Theta]_{0}$ versus water fraction, $[\Theta]_{0}$ : molar ellipticity at $f_{\mathrm{w}}=0 \%$. Copyright $(\mathrm{C} 2015$ Royal Society of Chemistry.

In order to answer this question, some control experiments were designed to construct the relationship between the twist angle of BN and the CD intensity. Firstly, two TPE moieties were removed from $(R)-\mathbf{1 7}$, and $(R)-18$ was obtained which was used to demonstrate whether TPE played some roles in the AACD effect. Figure 12 A showed that $(R)-18$ still exhibited the AACD effect even without TPE. The only difference was that transition $f_{\mathrm{w}}$ in $(R)$-18 was higher than $(R)-\mathbf{1 7}$, which should be attributed to the poorer solubility of $(R)-\mathbf{1 7}$ in THF and its aggregates were easier formed. This effect was supported by the dynamic light scattering (DLS) result. Above results suggested that AACD effect was caused by the change of $\mathrm{BN}$ within the process. After that, two naphthalene rings in $\mathrm{BN}$ was locked by a methylene group and $(R)-19$ was obtained. Although the AACD effect still existed, the transition $f_{\mathrm{w}}$ increased to $80 \%$. Methylene was replaced with a phosphate group as its stronger bond strength. It was expected that the two naphthalene rings could be fully locked in $(R)$ 20. Indeed, Figure $12 \mathrm{C}$ showed that there was no $\mathrm{CD}$ intensity change during the whole process of aggregation. Before the final conclusion was drawn, the authors proved that the aggregates were also formed in $(R)$-19 and $(R)-\mathbf{2 0}$ once the $f_{\mathrm{w}}$ was higher than 80\%, which indicated the AACD effect was partially suppressed in $(R)-\mathbf{1 9}$ but fully eliminated in $(R)-\mathbf{2 0}$. It was concluded that the AACD effect in axially chiral molecules, especially for BN derivatives, was caused by the change of twist angle between two adjacent naphthalene rings within the process of aggregation. It has been proved that the AACD effect widely existed in the BN based chiral molecules. ${ }^{28-31}$ But it was still under-explored whether other axially chiral molecules also showed the same AACD effect. 

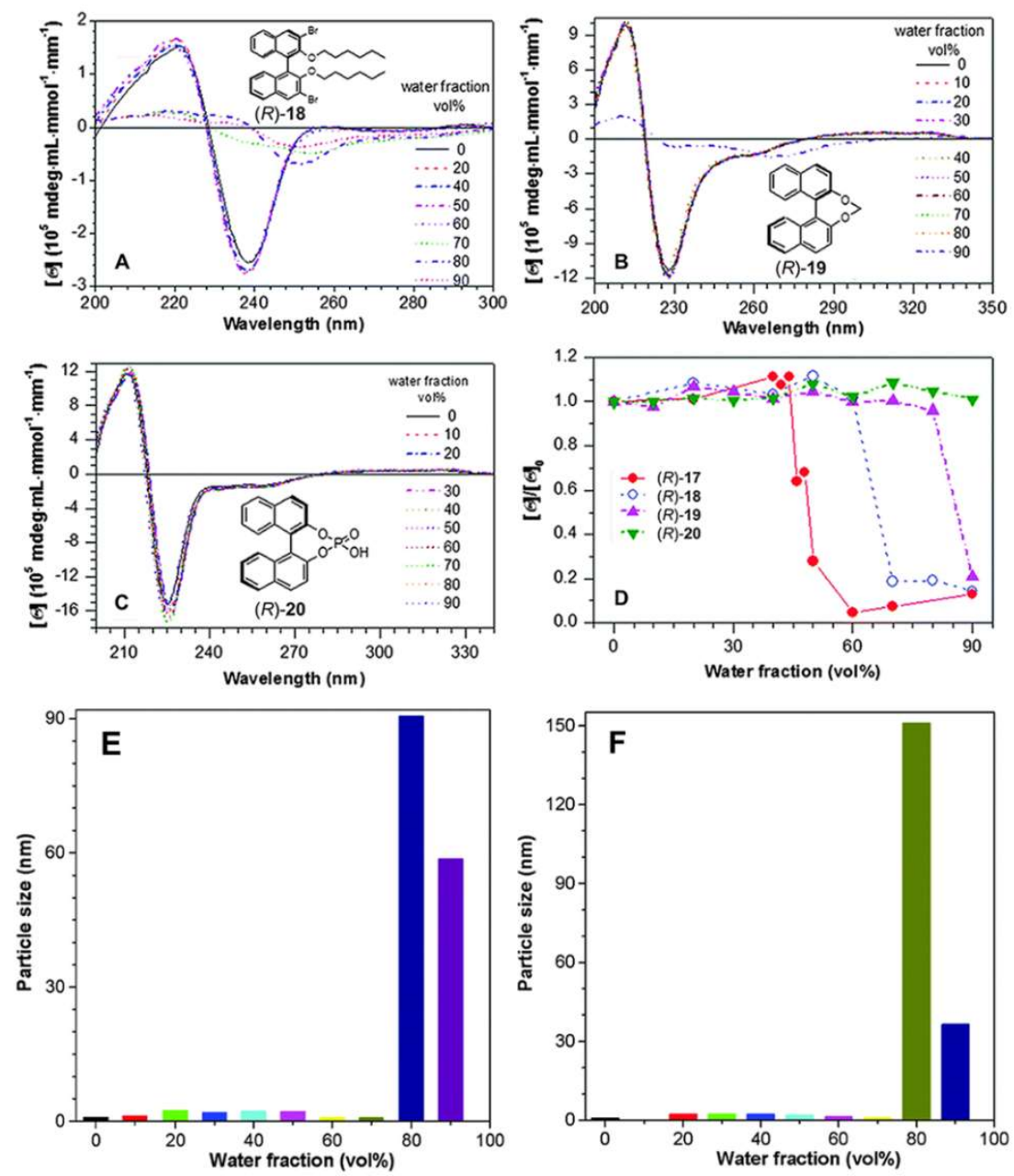

Figure 12. CD spectra of A) $(R)-\mathbf{1 8}, \mathrm{B})(R)-\mathbf{1 9}$ and C) $(R)-\mathbf{2 0}$ in THF/water mixtures with different $f_{\mathrm{w}}$. D) Plots of relative molar ellipticity of $(R)$ $18(@ 240 \mathrm{~nm}),(R)-19(@ 230 \mathrm{~nm})$ and $(R)-20(@ 230 \mathrm{~nm})$ versus $f_{\mathrm{w} .}$ DLS results of $\left.\mathrm{E}\right)(R)-19$ and $\left.\mathrm{F}\right)(R)-20$ in THF/water mixtures with $\operatorname{different} f_{\mathrm{w}}$. Copyright (C) 2015 Royal Society of Chemistry.

\section{CONCLUSION}

In summary, three systems were explored to study the relationship between aggregation and molecular chirality. The first one demonstrated how the chirality could be generated from the achiral system. The experimental results indicated that the aggregation effect played an important role in the spontaneous resolution. AICD effect was observed in some AIEgens decorated by chiral centers, it was proved that the chirality could transfer from chiral centers to AIEgens in the aggregate state but not the solution. Meanwhile, for those AIEgens, it was preliminarily concluded that the right-handed helical structures corresponded to a negative Cotton effect and vice versa. At last, AACD effect was discovered in axially chiral systems. Further studies suggested that the annihilation was caused by the change of twist angle between two adjacent naphthalene rings within the process of aggregation. The discovered relationship between chirality and aggregation brought significant insight into the origin of chirality. 


\section{ACKNOWLEDGMENT}

We are grateful for financial support from the National Science Foundation of China (21788102), the Research Grants Council of Hong Kong (16308016, N-HKUST604/14, C2014- 15G, C6009-17G and A-HKUST 605/16), the Innovation and Technology Commission (ITC-CNERC14SC01) and the Science and Technology Plan of Shenzhen (JCYJ20160229205601482 and JCY20170818113602462).

\section{REFERENCES}

[1] http,//blog.sciencenet.cn/blog-711486-658780.html.

[2] Glavin, D. P., Elsila, J. E., Burton, A. S., Callahan, M. P., Dworkin, J. P., Hilts, R. W. and Herd, C. D. K., "Unusual nonterrestrial L-proteinogenic amino acid excesses in the Tagish Lake meteorite," Meteorit. Planet. Sci. 47(8), 1347-1364 (2012).

[3] Luo, J., Xie, Z., Lam, W. Y., Cheng, L., Chen, H., Qiu, C., Kwok, H. S., Zhan, X. W., Liu, Y., Zhu, D. and Tang, B. Z., "Aggregation-induced emission of 1-methyl-1,2,3,4,5-pentaphenylsilole," Chem. Comm. 1740-1741 (2001).

[4] Mei, J., Leung, L. C., Kwok, T. K., Lam, W. Y. and Tang, B. Z., “Aggregation-Induced Emission, Together We Shine, United We Soar!” Chem. Rev. 115(21), 11718-940(2015).

[5] Ding, L., Lin, L., Liu, C., Li, H., Qin, A., Liu, Y., Song, L., Zhang, H. Tang, B. Z. and Zhao, Y., "Concentration effects in solid-state CD spectra of chiral atropisomeric compounds," New J. Chem. 35(9), 1781-1786 (2011).

[6] Li, D., Hu, R., Guo, D., Zang, Q., Li, J., Wang, Y., Zheng, Y., Tang, B. Z. and Zhang, H., "Diagnostic Absolute Configuration Determination of Tetraphenylethene Core-Based Chiral Aggregation-Induced Emission Compounds, Particular Fingerprint Bands in Comprehensive Chiroptical Spectroscopy," J. Phys. Chem. C 121(38), 20947-20954 (2017).

[7] Anuradha, La, D. D., Kobaisi, M. A. and Bhosale, S. V., "Right handed chiral superstructures from achiral molecules, self-assembly with a twist,” Sci. Rep. 5, 15652 (2015).

[8] Xiong, J. B., Feng, H. T., Sun, J. P., Xie, W. Z., Yang, D., Liu, M. and Zheng, Y. S., "The Fixed Propeller-Like Conformation of Tetraphenylethylene that Reveals Aggregation-Induced Emission Effect, Chiral Recognition, and Enhanced Chiroptical Property,” J. Am. Chem. Soc. 138(36), 11469-11472 (2016).

[9] Qu, H., Wang, Y., Li, Z., Wang, X., Fang, H., Tian, Z. and Cao, X., "Molecular Face-Rotating Cube with Emergent Chiral and Fluorescence Properties,” J. Am. Chem. Soc.139(50), 18142-18145(2017).

[10]Xue, S., Meng, L., Wen, R., Shi, L., Lam, W. Y., Tang, Z., Li, B. S. and Tang, B. Z., "Unexpected aggregation induced circular dichroism, circular polarized luminescence and helical assembly from achiral hexaphenylsilole (HPS)," RSC Adv. 7(40), 24841-24847(2017). 
[11]Liu, J., Su, H., Meng, L., Zhao, Y., Deng, H., Ng, C. Y., Lu, P., Faisal, M., Lam, W. Y., Huang, X., Wu, H., Wong, K. S. and Tang, B. Z., "What makes efficient circularly polarised luminescence in the condensed phase, aggregation-induced circular dichroism and light emission,” Chem. Sci. 3(9), 2737-2747(2012).

[12] Ng, C. Y., Li, H., Yuan, Q., Liu, J. Liu, C., Fan, X., Li, B. S. and Tang, B. Z., "Valine-containing silole, synthesis, aggregation-induced chirality, luminescence enhancement, chiral-polarized luminescence and self-assembled structures,” J. Mater. Chem. C 2(23), 4615-4621(2014).

[13]Li, H., Xue, S., Su, H., Shen, B., Cheng, Z., Lam, W. Y., Wong, K. S., Wu, H., Li, B. S. and Tang, B. Z., “Click Synthesis, Aggregation-Induced Emission and Chirality, Circularly Polarized Luminescence, and Helical SelfAssembly of a Leucine-Containing Silole," Small 12(47), 6593-6601(2016).

[14]Ng, C. Y., Liu, J., Su, H., Hong, Y., Li, H., Lam, W. Y., Wong, K. S. and Tang, B. Z., "Complexation-induced circular dichroism and circularly polarised luminescence of an aggregation-induced emission luminogen," J. Mater. Chem. C 2(1), 78-83(2014).

[15]Li, H., Zheng, X., Su, H., Lam, W. Y., Wong, K. S., Xue, S., Huang, X., Li, B. S. and Tang, B. Z, "Synthesis, optical properties, and helical self-assembly of a bivaline-containing tetraphenylethene," Sci. Rep. 6, 19277(2016).

[16]Li, H., Yuan, W., He, H., Cheng, Z., Fan, C., Yang, Y., Wong, K. S., Li, Y. and Tang, B. Z., “Circularly polarized luminescence and controllable helical self-assembly of an aggregation-induced emission luminogen,” Dyes. Pigm. $138,129-134(2017)$.

[17]Li, H., Cheng, J., Zhao, Y., Lam, W. Y., Wong, K. S., Wu, H., Li, B. S. and Tang, B. Z., "L-Valine methyl estercontaining tetraphenylethene, aggregation-induced emission, aggregation-induced circular dichroism, circularly polarized luminescence, and helical self-assembly," Mater. Horiz. 1(5), 518-521(2014).

[18]Li, H., Cheng, J., Deng, H., Zhao, E., Shen, B., Lam, W. Y., Wong, K. S., Wu, H., Li, B. S. and Tang, B. Z., "Aggregation-induced chirality, circularly polarized luminescence, and helical self-assembly of a leucinecontaining AIE luminogen,” J. Mater. Chem. C 3(10), 2399-2404(2015).

[19]Liu, X., Jiao, J., Jiang, X., Li, J., Cheng, Y. and Zhu, C., “A tetraphenylethene-based chiral polymer an AIE luminogen with high and tunable CPL dissymmetry factor," J. Mater. Chem. C 1, 4713-4719(2013).

[20]Liu, Q., Xia, Q., Wang, S., Li, B. S. and Tang, B. Z., "In situ visualizable self-assembly, aggregation induced emission and circularly polarized luminescence of tetraphenylethene and alanine based chiral polytriazole," J. Mater. Chem. C 6, 4807-4816(2018).

[21]Liang, K., Dong, L., Jin, N., Chen, D., Feng, X., Shi, J., Zhi, J., Tong, B. and Dong, Y., “The synthesis of chiral triphenylpyrrole derivatives and their aggregation-induced emission enhancement, aggregation-induced circular dichroism and helical self-assembly," RSC Adv. 6(28), 23420-23427(2016).

[22]Li, B. S., Wen, R., Xue, S., Shi, L., Tang, Z., Wang, Z. and Tang, B.Z., "Fabrication of circular polarized luminescent helical fibers from chiral phenanthro[9,10]imidazole derivatives," Mater. Chem. Front. 1(4), 646653(2017).

[23]Zhang, H., Li, H., Wang, J., Sun, J., Qin, A. and Tang, B.Z., "Axial chiral aggregation-induced emission luminogens with aggregation-annihilated circular dichroism effect," J. Mater. Chem. C 3(20), 5162-5166(2015). 
[24]Bari, L. D., Pescitelli, G. and Salvadori, P., “Conformational Study of 2,2'-Homosubstituted 1,1'-Binaphthyls by Means of UV and CD Spectroscopy," J. Am. Chem. Soc. 121, 7998-8004 (1999).

[25]Pescitelli, G., Bari, L. D and Berova, N., "Conformational aspects in the studies of organic compounds by electronic circular dichroism," Chem. Soc. Rev. 40, 4603-4625 (2011).

[26]Berova, N., Bari, L. D. and Pescitelli, G., "Application of electronic circular dichroism in configurational and conformational analysis of organic compounds," Chem. Soc. Rev. 36, 914-931 (2007).

[27] Pu, L. 1,1'-Binaphthyl-based Chiral Materials. Imperial College Press, London (2010).

[28] Wang, Z., Liu, S., Quan, Y. and Cheng, Y., "Tunable AICPL of (S)-Binaphthyl-Based Three-Component Polymers via FRET Mechanism,” Macromol. Rapid Commun. 38(14), 1700150 (2017).

[29]Li, N., Feng, H., Gong, Q., Wu, C., Zhou, H., Huang, Z., Yang, J., Chen, X. and Zhao, N., "BINOL-based chiral aggregation-induced emission luminogens and their application in detecting copper(ii) ions in aqueous media," J. Mater. Chem. C 3(43), 11458-11463(2015).

[30]Chen, S., Liu, W., Ge, Z., Zhang, W., Wang, K. and Hu, Z., "Synthesis and studies of axial chiral bisbenzocoumarins, Aggregation-induced emission enhancement properties and aggregation-annihilation circular dichroism effects," Spectrochim. Acta A 193, 141-146(2018).

[31] Yang, H. X., Xiang, K., Li, Y., Li, S. and Xu C., "Novel AIE luminogen containing axially chiral BINOL and tetraphenylsilole," J. Organomet. Chem. 801, 96-100(2016). 